\title{
QbD—Enabling Modern Product Development and Manufacturing
}

\author{
James K. Drennen III
}

Published online: 14 September 2010

(C) Springer Science+Business Media, LLC 2010

\begin{abstract}
Any pharmaceutical scientist who follows the current literature can likely cite numerous examples regarding the application of quality by design $(\mathrm{QbD})$ principles to the development and manufacturing of pharmaceuticals. Likewise, recent regulatory documents highlight the opportunity for advances in pharmaceutical product quality derived from $\mathrm{QbD}$ while providing the industry freedom to adopt modern methods of product development and manufacturing. The very recent (August 2010) draft Guidance for Industry: Residual Drug in Transdermal and Related Drug Delivery Systems gives direction to the manufacturers of transdermal and transmucosal systems, recommending that "an enhanced design and development approach - specifically quality by design $(\mathrm{QbD})$, as described in the International Conference on Harmonization (ICH) guidance for industry ( $Q 8(R 2)$ Pharmaceutical Development) — be used when developing and manufacturing TDDS [transdermal drug delivery systems], TMDS [transmucosal drug delivery systems], and topical patches." QbD is proposed for (1) the purpose of delivering "the optimum amount of drug across the skin while minimizing the amount of excess drug, thus resulting in the least possible amount of residual drug", and (2) enhancing the manufacturer's understanding of the product and process to assist in evaluating the effects of variability in both the raw materials and the manufacturing
\end{abstract}

J. K. Drennen III ( $\square)$

Duquesne University,

Pittsburgh, PA, USA

e-mail: jkdrennen@aol.com process on drug product quality, including performance characteristics.

The Journal of Pharmaceutical Innovation provides current material that will assist our pharmaceutical industry with the implementation of modern methods of product development and manufacturing, in concordance with the "guidance" of international regulatory bodies. In this issue, we present papers describing contemporary technologies and methodologies that can be applied as part of a $\mathrm{QbD}$ approach to pharmaceutical development and manufacturing. A case study discusses the application of multivariate tools to predict potential quality problems during the transition from the late stages of product development into early commercial manufacturing. A research article describes several methodologies for mapping design space. New technologies, including online dynamic image analysis and at-line powder avalanching, were evaluated in another research article for the purpose of optimizing a dry milling process. Additionally, an exceptional events management framework is described for the purpose of detecting, diagnosing, and mitigating exceptional events that are inherent to particulate processes that are prevalent in the pharmaceutical industry. Specifically, the method applies signed directed graph, qualitative trend analysis, and fast Fourier transforms to roller compaction for the purpose of detecting a variety of exceptional events.

As always, the Editorial Advisory Board looks forward to feedback from the readers of JPI, and we continue to seek quality manuscripts regarding innovative research and applied technologies within the pharmaceutical and biotechnology industries. 